Kragujevac Journal of Mathematics

Volume 40(1) (2016), Pages 61-72.

\title{
DIRECT LIMIT DERIVED FROM TWIST PRODUCT ON T-SEMIHYPERGROUPS
}

\author{
S. OSTADHADI-DEHKORDI
}

\begin{abstract}
The aim of this research work is to define a new class of hyperstructure that we call direct system. An important tool in the theory of homological algebra is the direct limit. We will present the construction of the direct limit of a direct system derived from $(\Delta, G)$-set on $\Gamma$-semihypergroups. Also, we prove the direct limit is unique up to isomorphism.
\end{abstract}

\section{INTRODUCTION}

The hypergroup notion was introduced in 1934 by a French mathematician F. Marty [9], at the $8^{\text {th }}$ Congress of Scandinavian Mathematicians. He published some notes on hypergroups, using them in different contexts: algebraic functions, rational fractions, non commutative groups. Algebraic hyperstructures are a suitable generalization of classical algebraic structures. In a classical algebraic structure, the composition of two elements is an element, while in an algebraic hyperstructure, the composition of two elements is a set. Since then, hundreds of papers and several books have been written on this topic, see [2-4].

Recently, the notion of $\Gamma$-hyperstructure introduced and studied by many researcher and represent an intensively was studied field of research, for example, see $[1,5,6,8]$. The concept of $\Gamma$-semihypergroups was introduced by Davvaz et al. $[1,8]$ and is a generalization of semigroups, a generalization of semihypergroups and a generalization of $\Gamma$-semigroups.

Key words and phrases. $\Gamma$-semihypergroup, left(right) $(\Delta, G)$-set, twist product, push out system, direct system, direct limit.

2010 Mathematics Subject Classification. 20N15.

Received: June 26, 2015.

Accepted: January 20, 2016. 
In this paper, we define the notion of left(right) $(\Delta, G)$-set, $\left(G_{1}, \Delta, G_{2}\right)$-biset, twist product, push out systems direct system and direct limits. Also, we prove that direct limit exists and unique.

\section{2. $\Gamma$-Semihypergroup And Twist Product}

In this section we present some notion of $\Gamma$-semihypergroup and introduce a relation denoted by $\rho^{*}$ which we shall use in order to define a new derived structure of $\Gamma$-semihypergroup that we called twist product. These definitions and results are necessary for the next section.

Definition 2.1. [8] Let $G$ and $\Gamma$ be nonempty sets and $\alpha: G \times G \longrightarrow P^{*}(G)$ be a hyperoperation, where $\alpha \in \Gamma$ and $P^{*}(G)$ be the set of all nonempty subset of $G$. Then, $G$ is called $\Gamma$-hypergroupoid.

For any two nonempty subset $G_{1}$ and $G_{2}$, we define

$$
G_{1} \alpha G_{2}=\bigcup_{g_{1} \in G_{1}, g_{2} \in G_{2}} g_{1} \alpha g_{2}, \quad G_{1} \alpha\{x\}=G_{1} \alpha x, \quad\{x\} \alpha G_{2}=x \alpha G_{2} .
$$

A $\Gamma$-hypergroupiod $G$ is a called $\Gamma$-semihypergroup if for all $x, y, z \in G$ and $\alpha, \beta \in \Gamma$, we have $(x \alpha y) \beta z=x \alpha(y \beta z)$, which means that

$$
\bigcup_{u \in x \alpha y} u \beta z=\bigcup_{v \in y \beta z} x \alpha v \text {. }
$$

Example 2.1. Let $\Gamma \subseteq \mathbb{N}$ be a nonempty set. We define

$$
x \widehat{\alpha} y=\{z \in \mathbb{N}: z \geq \max \{x, \alpha, y\}\},
$$

where $\alpha \in \widehat{\Gamma}=\{\widehat{\alpha}: \alpha \in \Gamma\}$ and $x, y \in \mathbb{N}$. Then, $\mathbb{N}$ is a $\widehat{\Gamma}$-semihypergroup.

Example 2.2. Let $\Gamma=\left\{\alpha_{1}, \alpha_{2}, \ldots, \alpha_{n}\right\}$. Then, we define hyperoperations $x \alpha_{k} y=$ $x y k \mathbb{Z}$. Hence $\mathbb{Z}$ is a $\Gamma$-semihypergroup.

Example 2.3. Let $G$ be a nonempty set and $\Gamma$ be a nonempty subset of $G$. We define $x \widehat{\alpha} y=\{x, \alpha, y\}$, where $\widehat{\Gamma}=\{\widehat{\alpha}: \alpha \in \Gamma\}$. Then, $G$ is a $\widehat{\Gamma}$-semihypergroup.

Example 2.4. Let $G$ be a group, $H$ be a normal subgroup of $G$ and $\Gamma \subseteq G$ be a nonempty subset. For all $g_{1}, g_{2} \in G$ and $\alpha \in \widehat{\Gamma}$, where $\widehat{\Gamma}=\{\widehat{\alpha}: \alpha \in \Gamma\}$ we define

$$
g_{1} \widehat{\alpha} g_{2}=g_{1} \alpha g_{2} H \text {. }
$$

Then, $G$ is a $\Gamma$-semihypergroup.

Let $G$ be a $\Gamma$-semihypergroup. Then, an element $e_{\alpha} \in G$ is called $\alpha$-identity if for every $x \in G$, we have $x \in e_{\alpha} \alpha x \cap x \alpha e_{\alpha}$ and $e_{\alpha}$ is called scaler $\alpha$-identity if $x=e_{\alpha} \alpha x=x \alpha e_{\alpha}$. We note that if for every $\alpha \in \Gamma, e$ is a scaler $\alpha$-identity, then $x \alpha y=x \beta y$, where $\alpha, \beta \in \Gamma$ and $x, y \in G$. Indeed,

$$
x \alpha y=(x \beta e) \alpha y=x \beta(e \alpha y)=x \beta y .
$$


Let $G$ be a $\Gamma$-semihypergroup and for every $\alpha \in \Gamma$ has an $\alpha$-identity. Then, $G$ is called a $\Gamma$-semihypergroup with identity.

Definition 2.2. Let $G$ be a $\Gamma$-semihypergroup and $\rho$ be an equivalence relation on $G$. Then, $\rho$ is called right regular if $x \rho y$ and $g \in G$ implies that for every $t_{1} \in x \alpha g$ there is $t_{2} \in y \alpha g$ such that $t_{1} \rho t_{2}$ and for every $s_{1} \in y \alpha g$ there is $s_{1} \in x \alpha g$ such that $s_{1} \rho s_{2}$. In a same way, we can define left regular relations.

Proposition 2.1. Let $G$ be a $\Gamma$-semihypergroup and $\rho$ be a regular relation on $G$. Then, $[G: \rho]=\{\rho(x): x \in G\}$ is a $\widehat{\Gamma}$-semihypergroup with respect to the following hyperoperation

$$
\rho(x) \widehat{\alpha} \rho(y)=\{\rho(z): z \in \rho(x) \alpha \rho(y)\},
$$

where $\widehat{\Gamma}=\{\widehat{\alpha}: \alpha \in \Gamma\}$.

Proof. The proof is straightforward.

Let $G$ be a $\Gamma$-semihypergroup and $\alpha \in \Gamma$. We define $x \circ y=x \alpha y$ for every $x, y \in G$. Hence $(G, \circ)$ becomes a semihypergroup, we denote this semihypergroup by $G[\alpha]$.

Definition 2.3. Let $G_{1}$ and $G_{2}$ be $\Gamma$-semihypergroup with identity. Then, a map $\varphi: G_{1} \longrightarrow G_{2}$ is called $\alpha$-homomorphism if $\varphi(x \alpha y)=\varphi(x) \alpha \varphi(y)$ and $\varphi\left(e_{\alpha}\right)=e_{\alpha}$ for every $x, y \in G_{1}$. If for every $\alpha \in \Gamma, \varphi$ is an $\alpha$-homomorphism, then $\varphi$ is called homomorphism.

Definition 2.4. Let $G$ be a $\Gamma$-semihypergroup with identity and $X, \Delta$ be nonempty sets. We say that $X$ is a left $(\Delta, G)$-set if for every $\delta \in \Delta$ there is an action $\delta: G \times X \longrightarrow X$ with the following properties:

$$
\begin{aligned}
\left(g_{1} \alpha g_{2}\right) \delta x & =g_{1} \alpha\left(g_{2} \delta x\right), \\
e_{\alpha} \alpha x & =x,
\end{aligned}
$$

for every $g_{1}, g_{2} \in G, \alpha \in \Gamma, x \in X$ and $\delta \in \Delta$.

In a same way, we can define a right $(\Delta, G)$-set. Let $G_{1}$ and $G_{2}$ be $\Gamma$-semihypergroups and $X$ be a nonempty set. Then, we say that $X$ is a $\left(G_{1}, \Delta, G_{2}\right)$ bisets if it is a left $\left(\Delta, G_{1}\right)$-set, right $\left(\Delta, G_{2}\right)$-set and

$$
\left(g_{1} \delta_{1} x\right) \delta_{2} g_{2}=g_{1} \delta_{1}\left(x \delta_{2} g_{2}\right),
$$

for every $\delta_{1}, \delta_{2} \in \Delta, g_{1} \in G_{1}, g_{2} \in G_{2}$ and $x \in X$.

A $\Gamma$-semihypergroup $G$ is called commutative when $x \alpha y=y \alpha x$, for every $x, y \in G$ and $\alpha \in \Gamma$. If $G$ is a commutative $\Gamma$-semihypergroup, then there is no distinction between a left and a right $(\Delta, G)$-set. A $(\Delta, G)$-left subset $Y$ of $X$ such that $Y \Delta X \subseteq Y$. A map $\varphi: X \longrightarrow Y$ from a left $(\Delta, G)$-set $X$ into a left $(\Delta, G)$-set $Y$ is called morphism if

$$
\varphi(g \delta x)=g \delta \varphi(x)
$$


for every $x \in X, \delta \in \Delta$ and $g \in G$. In a same way we can define a morphism of right $(\Delta, G)$-sets. An equivalence relation $\rho$ on left $(\Delta, G)$-set $X$ is called congruence, if for every $x, y \in X, \delta \in \Delta$ and $g \in G$

$$
x \rho y \Longrightarrow(g \delta x) \rho(g \delta y) .
$$

The quotient $[X: \rho]$ is a left $(\widehat{\Delta}, G)$-set by following operation:

$$
g \widehat{\delta}(\rho(x))=\rho(g \delta x)
$$

where $\widehat{\Delta}=\{\widehat{\delta}: \delta \in \Delta\}$. The map $\pi: X \longrightarrow[X: \rho]$ defined by $\pi(x)=\rho(x)$, for every $x \in X$ is a morphism.

Example 2.5. Let $G$ be a $\Gamma$-semihypergroup and $G_{1}$ be a $\Gamma$-subsemihypergroup of $G$. Then, $G_{1}$ is a left $\left(\Gamma, G_{1}\right)$-set in the obvious way.

Example 2.6. Let $\rho$ be a left regular relation on $\Gamma$-semihypergroup $G$. Then, there is a well-defined action of $G$ on $[G: \rho]$ given by $g \widehat{\alpha}(\rho(x))=\rho(g \alpha x)$, where $\widehat{\alpha} \in \widehat{\Gamma}$ and $x \in G$ such that $\widehat{\Gamma}=\{\widehat{\alpha}: \alpha \in \Gamma\}$. Hence, with this definition $[G: \rho]$ is a left $(\widehat{\Gamma}, G)$-set.

It is easy to see that the cartesian product $X \times Y$ of a left $\left(\Delta, G_{1}\right)$-set $X$ and a right $\left(\Delta, G_{2}\right)$-set $Y$ becomes $\left(G_{1}, \widehat{\Delta}, G_{2}\right)$-biset if we make the obvious definition

$$
g_{1} \widehat{\delta}_{1}(x, y)=\left(g_{1} \delta_{1} x, y\right), \quad(x, y) \widehat{\delta}_{2} g_{2}=\left(x, y \delta_{2} g_{2}\right)
$$

where $\widehat{\delta}_{1}, \widehat{\delta}_{2} \in \widehat{\Delta}, x \in X, y \in Y$ and $g_{1} \in G_{1}, g_{2} \in G_{2}$.

Let $X, Y$ and $Z$ be $\left(G_{1}, \Delta, G_{2}\right)$-biset, $\left(G_{2}, \Delta, G_{3}\right)$-biset, and $\left(G_{1}, \Delta, G_{3}\right)$-biset (respectively). Then, the cartesian product $X \times Y$ is $\left(G_{1}, \Delta, G_{3}\right)$-biset. A map $\varphi: X \times Y \longrightarrow Z$ is called $\delta$-bimap if

$$
\varphi\left(x \delta g_{2}, y\right)=\varphi\left(x, g_{2} \delta y\right)
$$

where $x \in X, y \in Y, z \in Z, g_{2} \in G_{2}$ and $\delta \in \Delta$.

Definition 2.5. [7] A pair $(P, \psi)$ consisting of $\left(G_{1}, \Delta, G_{3}\right)$-biset $P$ and a $\delta$-bimap $\psi: X \times Y \longrightarrow P$ will be called a twist product of $X$ and $Y$ over $G_{2}$ if for every $\left(G_{1}, \Delta, G_{3}\right)$-biset $Z$ and for every bimap $\omega: X \times Y \longrightarrow Z$ there exists a unique bimap $\bar{\omega}: P \longrightarrow Z$ such that $\bar{\omega} \circ \psi=\omega$.

Suppose that $\rho$ is an equivalence relation on $X \times Y$ as follows:

$$
\rho=\left\{((x \delta g, y),(x, g \delta y)), x \in X, y \in Y, g \in G_{2}\right\} .
$$

Let us define $X \ominus Y$ to be $\left[X \times Y: \rho^{*}\right]$, where $\rho^{*}$ is a transitive closure of $\rho$. We denote a typical element $\rho^{*}(x, y)$ by $x \ominus y$. By definition of $\rho^{*}$, we have $x \delta g \ominus y=x \ominus g \delta y$, where $\delta \in \Delta$.

Proposition 2.2. [7] Let $X$ and $Y$ be $\left(G_{1}, \Delta, G_{2}\right)$-biset and $\left(G_{2}, \Delta, G_{3}\right)$-biset, respectively. Then, two elements $x \ominus y$ and $x^{\prime} \ominus y^{\prime}$ are equal if and only if $(x, y)=\left(x^{\prime}, y^{\prime}\right)$ or 
there exist $x_{1}, x_{2}, \ldots, x_{n-1}$ in $X, y_{1}, y_{2}, \ldots, y_{n-1} \in Y, g_{1}, g_{2}, \ldots, g_{n}, h_{1}, h_{2}, \ldots, h_{n-1} \in$ $G_{2}$ and $\delta \in \Delta$ such that

$$
\begin{aligned}
x & =x_{1} \delta g_{1}, & g_{1} \delta y & =h_{1} \delta y_{1}, \\
x_{1} \delta h_{1} & =x_{2} \delta g_{2}, & g_{2} \delta y_{1} & =h_{2} \delta y_{2}, \\
& \vdots & & \vdots \\
x_{i} \delta g_{i} & =x_{i+1} \delta g_{i+1}, & g_{i+1} \delta y_{i} & =h_{i+1} \delta y_{i+1}, \\
& \vdots & & \vdots \\
x_{n-1} \delta h_{n-1} & =x^{\prime} \delta g_{n}, & g_{n} \delta y_{n-1} & =y^{\prime} .
\end{aligned}
$$

Theorem 2.1. [7] Let $X$ and $Y$ be $\left(G_{1}, \Delta, G_{2}\right)$-biset and $\left(G_{2}, \Delta, G_{3}\right)$-biset. Then, $(X \ominus Y, \pi)$ is a twist product of $X$ and $Y$ over $G_{2}$.

Proof. It is easy to see that $\pi: X \times Y \longrightarrow X \ominus Y$ is a $\delta$-bimap such that $\pi(x, y)=x \ominus y$. Let $\omega: X \times Y \longrightarrow Z$, where $Z$ is a $\left(G_{1}, \Delta, G_{3}\right)$-biset and $\omega$ is a $\delta$-bimap. We define $\bar{\omega}: X \ominus Y \longrightarrow Z$ by

$$
\bar{\omega}(x \ominus y)=\omega(x, y) .
$$

Let $x \ominus y=x^{\prime} \ominus y^{\prime}$. By 2.2, we have

$$
\omega(x, y)=\omega\left(x_{1} \delta g_{1}, y\right)=\omega\left(x_{1}, g_{1} \delta y\right)=\omega\left(x_{1}, g_{1} \delta h_{1}\right)=\cdots=\omega\left(x^{\prime}, y^{\prime}\right) .
$$

Hence $\bar{\omega}(x \ominus y)=\bar{\omega}\left(x^{\prime} \ominus y^{\prime}\right)$. It is easy to see that $\bar{\omega}$ is a $\delta$-bimap, $\bar{\omega} \circ \pi=\omega$ and $\bar{\omega}$ is unique with respect.

Theorem 2.2. Let $X$ and $Y$ be $\left(G_{1}, \Delta, G_{2}\right)$-biset and $\left(G_{2}, \Delta, G_{3}\right)$-biset. Then the twist product $X$ and $Y$ over $G_{2}$ is unique up to isomorphism.

Proof. Suppose that $(P, \psi)$ and $\left(P^{\prime}, \psi^{\prime}\right)$ are twist product of $X$ and $Y$ over $G_{2}$. By definition we find a unique $\overline{\psi^{\prime}}: P \longrightarrow P^{\prime}$ and $\bar{\psi}: P^{\prime} \longrightarrow P$ such that $\psi \circ \overline{\psi^{\prime}}=\psi^{\prime}$ and $\bar{\psi}: P^{\prime} \longrightarrow P \operatorname{such} \psi^{\prime} \circ \bar{\psi}=\psi$. Since $\psi \circ \overline{\psi^{\prime}} \circ \bar{\psi}=\psi$, we have $\overline{\psi^{\prime}} \circ \bar{\psi}=I d_{P^{\prime}}$. By a similar argument $\bar{\psi} \circ \overline{\psi^{\prime}}=I d_{P}$.

We can generalize the notion of twist product three bisets. Let $X, Y, Z$ and $W$ be $\left(G_{1}, \Delta, G_{2}\right)$-biset, $\left(G_{2}, \Delta, G_{3}\right)$-biset, $\left(G_{3}, \Delta, G_{4}\right)$-biset and $\left(G_{1}, \Delta, G_{4}\right)$-biset. Then, a map $\varphi: X \times Y \times Z \longrightarrow Z$ is called $\delta$-trimap if for $x \in X, y \in Y$ and $z \in Z$ and $g_{2} \in G_{2}, g_{3} \in G_{3}$ and $\delta \in \Delta$

$$
\varphi\left(x \delta g_{2}, y, z\right)=\varphi\left(x, g_{2} \delta y, z\right), \quad \varphi\left(x, y \delta g_{3}, z\right)=\varphi\left(x, y, g_{3} \delta z\right) .
$$

A pair $(P, \psi)$, where $P$ is a $\left(G_{1}, \Delta, G_{4}\right)$-biset and $\psi: X \times Y \times Z \longrightarrow P$ is a $\delta$-trimap is said to be twist if for every $\left(G_{1}, \Delta, G_{4}\right)$-biset $W$ and every $\delta$-trimap $\phi: X \times Y \times Z \longrightarrow W$ there is a unique $\bar{\phi}: P \longrightarrow W$ such that $\bar{\phi} \circ \psi=\phi$. A similar argument shows that $X \ominus(Y \ominus Y)$, together with the obvious trimap $(x, y, z) \longrightarrow x \ominus(y \ominus z)$ is also a twist product of $X, Y$ and $Z$. 
Proposition 2.3. [7] Let $X, Y, Z$ be $\left(G_{1}, \Delta, G_{2}\right)$-biset, $\left(G_{2}, \Delta, G_{3}\right)$-biset, $\left(G_{3}, \Delta, G_{4}\right)$ biset, respectively. Then, $X \ominus(Y \ominus Z) \cong(X \ominus Y) \ominus Z$.

Suppose that $\varphi: X_{1} \longrightarrow X_{2}$ is a morphism and

$$
\operatorname{kerl} \varphi:\left\{(a, b) \in X_{1} \times X_{1}: \varphi(a)=\varphi(b)\right\} .
$$

This relation on $X_{1}$ is an equivalence relation and is called kernel of $\varphi$.

Theorem 2.3. Let $G$ be a $\Gamma$-semihypergroup, $X_{1}, X_{2}$ be left $(\Delta, G)$-sets, $\varphi: X_{1} \longrightarrow$ $X_{2}$ be a morphism and $\rho \subseteq \operatorname{kerl} \varphi$ be a congruence relation on $X_{1}$. Then, $\left[X_{1}: \rho\right]$ is a $(\widehat{\Delta}, G)$-set, where $\widehat{\Delta}=\{\widehat{\delta}: \delta \in \Delta\}$ and there is a monomorphism $\widehat{\varphi}:\left[X_{1}: \rho\right] \longrightarrow \operatorname{Im} \varphi$. Proof. It is easy to see that $\left[X_{1}: \rho\right]$ is a $(\widehat{\Delta}, G)$-set. We Define $\widehat{\varphi}:\left[X_{1}: \rho\right] \longrightarrow X_{2}$ by

$$
\widehat{\varphi}(\rho(x))=\varphi(x) \text {. }
$$

Let $\rho(a)=\rho(b)$. Then,

$$
(a, b) \in \rho \Longrightarrow(a, b) \in \operatorname{kerl} \varphi \Longrightarrow \varphi(a)=\varphi(b) .
$$

This implies that $\widehat{\varphi}$ is well-defined. If $g \in G$ and $\rho(a) \in\left[X_{1}: \rho\right]$, then

$$
\widehat{\varphi}(g \widehat{\delta} \rho(a))=\widehat{\varphi}(\rho(g \delta a))=\varphi(g \delta a)=g \delta \varphi(a)=g \delta \widehat{\varphi}(\rho(a)) .
$$

Hence $\widehat{\varphi}$ is a morphism.

Proposition 2.4. Let $\rho_{1}$ and $\rho_{2}$ be congruence relations on $(\Delta, G)$-set $X$ such that $\rho_{1} \subseteq \rho_{2}$. Then,

$$
\left[\rho_{2}: \rho_{1}\right]=\left\{\left(\rho_{1}(a), \rho_{1}(b)\right) \in\left[X: \rho_{1}\right] \times\left[X: \rho_{2}\right]:(a, b) \in \rho_{2}\right\},
$$

is a congruence relation on $\left[X: \rho_{1}\right]$ and

$$
\left[\left[X: \rho_{1}\right]:\left[\rho_{2}: \rho_{1}\right]\right] \simeq\left[X: \rho_{2}\right] .
$$

Proof. The proof is straightforward.

\section{DiRECT Limit}

In this section we introduce a non additive version of direct limit that is important in homological algebra. We prove that the direct limit exists and is unique.

Let $(J, \leq)$ be a partially ordered set and $\left\{X_{j}\right\}_{j \in J}$ be a collection of $\left(G_{1}, \Delta, G_{2}\right)$ bisets and for all $i, j \in J$ such that $i \leq j$, there is a morphism $\omega_{i j}: X_{i} \longrightarrow X_{j}$ with the following properties:

1) $\omega_{i i}=I_{X_{i}}$

2) $\omega_{i j} \circ \omega_{j k}=\omega_{i k}$.

Then, we say that $\left(X_{i}, \omega_{i j}\right)$ is a direct system of $\left(G_{1}, \Delta, G_{2}\right)$-bisets.

We say that a $\left(G_{1}, \Delta, G_{2}\right)$-biset $X$ is called direct limit of this direct system if there exist morphisms $\omega_{i}: X_{i} \longrightarrow X$ such that $\omega_{j} \circ \omega_{i j}=\omega_{i}$ and if there exists a $\left(G_{1}, \Delta, G_{2}\right)$ - biset $Y$ has the property that there exist a morphism $\lambda_{i}: X_{i} \longrightarrow Y$ such that $\lambda_{j} \circ \alpha_{i j}=\lambda_{i}$ with $i \leq j$ and $\alpha_{i j}: X_{i} \longrightarrow X_{j}$ is a morphism, then there is a unique morphism $\lambda: X \longrightarrow Y$ such that $\lambda \circ \omega_{i}=\lambda_{i}$. 
Theorem 3.1. Let $\left(X_{i}, \omega_{i j}\right)$ be a direct system. Then, the direct limit exist.

Proof. Suppose that $\left(X_{i}, \omega_{i j}\right)$ is a direct system. Without loses of generality we suppose that the sets $X_{i}$ are pairwise disjoint. Let $D=\bigcup_{i \in I} X_{i}$ and $\theta^{*}$ be equivalence relation generated by the following relation:

$$
d_{1} \theta d_{2} \Longleftrightarrow \text { there exists } i \leq j, \quad d_{1} \in X_{i}, \quad \omega_{i j}\left(d_{1}\right)=d_{2},
$$

where $d_{1}, d_{2} \in D, i, j \in I$. We prove that $\left[D: \theta^{*}\right]$ is a direct limit. To see this, we define a morphism $\omega_{i}: X_{i} \longrightarrow\left[D: \theta^{*}\right]$, for each $i \in I$ by

$$
\omega_{i}\left(x_{i}\right)=\theta^{*}\left(x_{i}\right)
$$

where $x_{i} \in X_{i}$. We have

$$
\omega_{j} \circ \omega_{i j}\left(x_{i}\right)=\theta^{*}\left(\omega_{i j}\left(x_{i}\right)\right)=\theta^{*}\left(x_{i}\right),
$$

for every $x_{i} \in X_{i}$. Let $Y$ be a $\left(G_{1}, \Delta, G_{2}\right)$-biset and $\lambda_{i}: X_{i} \longrightarrow Y$ be a morphism such that $\lambda_{j} \circ \alpha_{i j}=\lambda_{i}$. We define a morphism $\varphi: D \longrightarrow Y$ by

$$
\varphi\left(x_{i}\right)=\lambda_{i}\left(x_{i}\right), \quad x_{i} \in X_{i}, \quad i \in I .
$$

Let $d_{1} \theta d_{2}$. Then, there are $i, j \in I$ and $\omega_{i j}$ such that $\omega_{i j}\left(d_{1}\right)=d_{2}$. This implies that

$$
\varphi\left(d_{2}\right)=\varphi\left(\omega_{i j}\left(d_{1}\right)\right)=\lambda_{j}\left(\omega_{i j}\left(d_{1}\right)\right)=\lambda_{i}\left(d_{1}\right)=\varphi\left(d_{1}\right) .
$$

Hence $\left(d_{1}, d_{2}\right) \in \operatorname{ker} \varphi$ and by Proposition 2.4 , there exists morphism $\widehat{\varphi}:\left[D: \theta^{*}\right] \longrightarrow Y$ defined by

$$
\widehat{\varphi}\left(\theta^{*}\left(x_{i}\right)\right)=\varphi\left(x_{i}\right),
$$

where $x_{i} \in X_{i}, i \in I$. Also, $\widehat{\varphi} \circ \omega_{i}\left(x_{i}\right)=\widehat{\varphi}\left(\theta^{*}\left(x_{i}\right)\right)=\lambda_{i}\left(x_{i}\right)$. This implies that $\widehat{\varphi} \circ \omega_{i}=\lambda_{i}$. If $\psi$ is a morphism with the same properties, then for every $x_{i} \in X_{i}$ and $i \in I$,

$$
\psi\left(\theta^{*}\left(x_{i}\right)\right)=\psi\left(\omega_{i}\left(x_{i}\right)\right)=\lambda_{i}\left(x_{i}\right)=\widehat{\varphi}\left(\theta^{*}\left(x_{i}\right)\right) .
$$

Therefore, $\widehat{\varphi}=\psi$. This completes the proof.

Proposition 3.1. The direct limit of direct system $\left(X_{i}, \omega_{i j}\right)_{i, j \in I}$ is unique up to isomorphism.

Proof. Suppose that $X$ and $Y$ are direct limits of direct system $\left(X_{i}, \omega_{i j}\right)_{i, j \in I}$. By definition we have a unique $\lambda: X \longrightarrow Y$ and $\lambda^{\prime}: Y \longrightarrow X$ such that $\omega_{i} \circ \lambda=\lambda_{i}$ and $\lambda_{i} \circ \lambda^{\prime}=\omega_{i}$. Hence

$$
\begin{aligned}
& \omega_{i} \circ\left(\lambda \circ \lambda^{\prime}\right)=\left(\omega_{i} \circ \lambda\right) \circ \lambda^{\prime}=\lambda_{i} \circ \lambda^{\prime}=\omega_{i}, \\
& \lambda_{i} \circ\left(\lambda^{\prime} \circ \lambda\right)=\lambda_{i} .
\end{aligned}
$$

Therefore, $\lambda \circ \lambda^{\prime}=I_{X}$ and $\lambda^{\prime} \circ \lambda=I_{Y}$ and so $X \cong Y$.

Proposition 3.2. Let $\left(X_{n}, \omega_{n}\right)$ be a direct system and $\left[D: \theta^{*}\right]$ be the direct limit of the this direct system. Then, the map $\beta_{n}: X_{n} \longrightarrow\left[D: \theta^{*}\right]$ is one to one if and only if the maps $\omega_{n}$ are one to one. 
Proof. Suppose that all maps $\omega_{n}: X_{n} \longrightarrow X_{n+1}$ are one to one and $\beta_{m}\left(a_{m}\right)=\beta_{m}\left(b_{m}\right)$. This implies that $\theta^{*}\left(a_{m}\right)=\beta^{*}\left(b_{m}\right)$. Hence

$$
\text { there exists } x_{1}, x_{2}, \ldots, x_{n} \in D: a_{m}=x_{1}, b_{m}=x_{n} \text { and } x_{i} \theta x_{i+1} \text {. }
$$

By the definition of $\theta$, since every $\omega_{n}$ is one to one it follows that $a_{m}=b_{m}$. This implies that $\beta_{n}: X_{n} \longrightarrow X_{n+1}$ is one to one. Conversely, suppose that for some $n, \omega_{n}$ is not one to one and $\beta_{n}$ is one to one. Hence for some $a_{n} \neq b_{n}$ we have $\omega_{n}\left(a_{n}\right)=\omega_{n}\left(b_{n}\right)$. By definition $a_{n} \theta b_{n}$ and $\theta^{*}\left(a_{n}\right)=\theta^{*}\left(b_{n}\right)$ and so $\beta^{*}\left(a_{n}\right)=\beta^{*}\left(b_{n}\right)$. That is contradiction.

Proposition 3.3. Let $\left(X_{i}, \omega_{i j}\right)$ be a direct system $\left(G_{2}, \Delta, G_{3}\right)$-biset, $D$ be the direct limit of this direct system and $H_{1}, H_{2}$ be $\left(G_{1}, \Delta, G_{2}\right),\left(G_{3}, \Delta, G_{4}\right)$-biset, respectively. Then, $H_{1} \ominus D \ominus H_{2}$ is the direct limit of direct system $\left(H_{1} \ominus X_{i} \ominus H_{2}, I_{H_{1}} \ominus \omega_{i j} \ominus I_{H_{2}}\right)$.

Proof. Suppose that

$$
\begin{gathered}
I_{H_{1}} \ominus \omega_{i j} \ominus I_{H_{2}}: H_{1} \ominus X_{i} \ominus H_{2} \longrightarrow H_{1} \ominus X_{j} \ominus H_{2}, \\
I_{H_{1}} \ominus \omega_{i} \ominus I_{H_{2}}: H_{1} \ominus X_{i} \ominus H_{2} \longrightarrow H_{1} \ominus D \ominus H_{2} .
\end{gathered}
$$

Obviously,

$$
\left(I_{H_{1}} \ominus \omega_{i} \ominus I_{H_{2}}\right) \circ\left(I_{H_{1}} \ominus \omega_{i j} \ominus I_{H_{2}}\right)=I_{H_{1}} \ominus \omega_{i} \ominus I_{H_{2}},
$$

for $i \leq j$. Let $Q$ be a $\left(G_{1}, \Delta, G_{4}\right)$-biset and $\sigma_{i}: H_{1} \ominus X_{i} \ominus H_{2} \longrightarrow Q$ such that $\left(I_{H_{1}} \ominus \omega_{i j} \ominus I_{H_{2}}\right) \circ \sigma_{j}=\sigma_{i}$, for all $i \leq j$ and $T$ is the disjoint union $X_{i}$ and $\theta^{*}$ is the equivalence relation generated by the following relation:

$$
d_{1} \theta d_{2} \Longleftrightarrow \text { there exists } i, j \in I \omega_{i j}\left(d_{2}\right)=d_{1} .
$$

We know that $\omega_{i}\left(x_{i}\right)=\theta^{*}\left(x_{i}\right)$ for all $x_{i} \in X_{i}$. We define $\mu: H_{1} \times T \times H_{2} \longrightarrow Q$ by

$$
\mu\left(h_{1}, t_{i}, h_{2}\right)=\sigma_{i}\left(h_{1} \ominus t_{i} \ominus h_{2}\right) .
$$

We have

$$
\begin{aligned}
\mu\left(h_{1}, \omega_{i j}\left(t_{i}\right), h_{2}\right) & =\sigma_{i}\left(h_{1} \ominus \omega_{i j}\left(t_{i}\right) \ominus h_{2}\right) \\
& =\sigma_{j}\left(I_{H_{2}} \ominus \omega_{i j} \ominus I_{H_{2}}\right)\left(h_{1} \ominus t_{i} \ominus h_{2}\right) \\
& =\sigma_{i}\left(h_{1} \ominus t_{i} \ominus h_{2}\right) \\
& =\mu\left(h_{1}, t_{i}, h_{2}\right) .
\end{aligned}
$$

Hence $\mu$ induces a map $\widehat{\mu}: H_{1} \times T \times H_{2} \longrightarrow Q$ defined by

$$
\widehat{\mu}\left(h_{1}, \theta^{*}\left(t_{i}\right), h_{2}\right)=\sigma_{i}\left(h_{1} \ominus t_{i} \ominus h_{2}\right) .
$$

For all $g_{2} \in G_{2}$ and $t_{i} \in T$ we have

$$
\widehat{\mu}\left(h_{1} \delta g_{2}, \theta^{*}\left(t_{i}\right), h_{2}\right)=\sigma_{i}\left(h_{1} \delta g_{2} \ominus t_{i} \ominus h_{2}\right)=\sigma_{i}\left(h_{1} \ominus g_{2} \delta x_{i} \ominus h_{2}\right)=\widehat{\mu}\left(h_{1}, g_{2} \theta^{*}\left(t_{i}\right), h_{2}\right),
$$

and similarly, for every $g_{3} \in G_{3}$ we have

$$
\widehat{\mu}\left(h_{1}, \theta^{*}\left(t_{i}\right), g_{3} \delta h_{2}\right)=\widehat{\mu}\left(h_{1}, \theta^{*}\left(t_{i}\right) \delta g_{3}, h_{2}\right) .
$$


Hence $\widehat{\mu}$ induces a map $\xi: H_{1} \ominus T \ominus H_{2} \longrightarrow Q$ given by

$$
\xi\left(h_{1} \ominus \theta^{*}\left(t_{i}\right) \ominus h_{2}\right)=\sigma_{i}\left(h_{1} \ominus t_{i} \ominus h_{2}\right) .
$$

It is easy to see that $\xi$ is morphism and $\left(I_{H_{1}} \omega_{i} \ominus I_{H_{2}}\right) \circ \xi=\sigma_{i}$ and $\xi$ is unique. This completes the proof.

Suppose that $X_{1}, X_{2}, X_{3}, X_{4}$ are $(\Delta, G)$-sets and $\varphi_{i}: X_{1} \longrightarrow X_{i}, \psi_{j}: X_{j} \longrightarrow X_{4}$ are morphisms for $2 \leq i \leq 3$ and $2 \leq j \leq 3$ such that $\psi_{2} \circ \varphi_{1}=\psi_{3} \circ \varphi_{2}$. If there are $\psi_{2}^{\prime}: X_{2} \longrightarrow X_{4}^{\prime}$ and $\psi_{3}^{\prime}: X_{3} \longrightarrow X_{4}^{\prime}$, such that $\psi_{3}^{\prime} \circ \varphi_{2}=\psi_{2}^{\prime} \circ \varphi_{1}$, then there is a morphism $\lambda: X_{4} \longrightarrow X_{4}^{\prime}$ such that $\lambda \circ \psi_{2}=\psi_{2}^{\prime}$ and $\lambda \circ \psi_{3}=\psi_{3}^{\prime}$. A system $\left[X_{i}, \varphi_{j}, \psi_{k}\right]$ for $1 \leq i \leq 4,1 \leq j \leq 2,1 \leq k \leq 2$ is called push out.

Proposition 3.4. Let $X_{1}, X_{2}, X_{3}$ be $(\Delta, G)$-sets, $\varphi_{1}: X_{1} \longrightarrow X_{2}$ and $\varphi_{2}: X_{1} \longrightarrow X_{3}$ be morphisms. Then, there is a push out system and $x_{2} \in X_{2}, x_{3} \in X_{3}, \psi_{2}\left(x_{2}\right)=$ $\psi_{3}\left(x_{3}\right)$ implies that $x_{2} \in \operatorname{Im} \varphi_{1}$.

Proof. Suppose that $\rho$ is a following relation on $X=X_{1} \cup X_{2} \cup X_{3}$ of $\operatorname{disjoint}(\Delta, G)$ sets.

$$
x \rho y \Longleftrightarrow x \in X_{1} \text { and } y=\varphi_{1}(x) \text { or } x \in X_{1} \text { and } \varphi_{2}(x)=y .
$$

Let $\rho^{*}$ be equivalence relation generated by $\rho$ and $\left[X: \rho^{*}\right]$ be the quotient set on $X$ by $\rho^{*}$. We define $\psi_{2}: X_{2} \longrightarrow\left[X: \rho^{*}\right]$ and $\psi_{3}: X_{3} \longrightarrow\left[X: \rho^{*}\right]$ by

$$
\psi_{2}\left(x_{2}\right)=\rho^{*}\left(x_{2}\right), \psi_{3}\left(x_{3}\right)=\rho^{*}\left(x_{3}\right) .
$$

It is easy to see that $\left[X, X_{i}, \varphi_{j}, \varphi_{k}\right]$ is a push out system.

Let $\psi_{2}\left(x_{2}\right)=\psi_{3}\left(x_{3}\right)$, then $\rho^{*}\left(x_{2}\right)=\rho^{*}\left(x_{3}\right)$. This implies that there are $a_{1}, a_{2}, \ldots, a_{n} \in X$ such that $a_{1}=x_{2}, a_{n}=x_{3}$ and $a_{i} \rho a_{i+1}$. Hence $a_{i} \in X_{1}$ and $\varphi_{1}\left(a_{i}\right)=a_{i+1}$ or $\varphi_{2}\left(a_{i}\right)=a_{i+1}$. This implies that $x_{2} \in \operatorname{Im} \varphi_{1}$.

Let $G_{1}$ be a $\Gamma$-subsemihypergroup of $G, X_{1}$ and $X_{2}$ be a $\left(\Delta, G_{1}\right)$-set and a $(\Delta, G)$ set, respectively and $X=X_{2} \ominus G$, and $\varphi: X_{1} \longrightarrow X_{2}$ be a morphism on $G_{1}$. We define the following relation on $X$ as follows:

$\left(d_{1} \ominus g_{1}\right) \xi\left(d_{2} \ominus g_{2}\right) \Longleftrightarrow$ there exists $t_{1}, t_{2} \in X_{1}, \varphi\left(t_{1}\right)=d_{1}, \varphi\left(t_{2}\right)=d_{2}, t_{1} \delta g_{1}=t_{2} \delta g_{2}$.

Suppose that $\xi^{*}$ is an equivalence relation generated by $\xi$. Hence $\left[X \ominus G: \xi^{*}\right]$ is a $(\Delta, G)$-set and is called extension of $X$ by $G$.

Proposition 3.5. Let $G_{1}$ be a $\Gamma$-subsemihypergroup of $G, X_{1}$ be a left $(\Delta, G)$-set, $X_{2}$ be a left $\left(\Delta, G_{1}\right)$-set and $\varphi: X_{1} \longrightarrow X_{2}$ be a morphism and $T=\left[X_{2} \ominus G: \xi^{*}\right]$, where $\Delta \subseteq \Gamma$. Then, $X_{1} \ominus G, X_{2} \ominus G, X_{1}, T$, where $\varphi \ominus I: X_{1} \ominus G \longrightarrow X_{2} \ominus G, \psi$ : $X_{1} \ominus G \longrightarrow X_{1}, \beta: X_{1} \longrightarrow T$ and $\pi: X_{2} \ominus G \longrightarrow T$ defined as follows:

$$
\begin{aligned}
& \varphi \ominus I\left(x_{1} \ominus g\right)=\varphi\left(x_{1}\right) \ominus g, \psi\left(x_{1} \ominus g\right)=x_{1} \delta g, \\
& \beta\left(x_{1}\right)=\xi^{*}\left(\varphi\left(x_{1}\right) \ominus e_{\delta}\right), \pi\left(x_{2} \ominus g\right)=\xi^{*}\left(x_{2} \ominus g\right),
\end{aligned}
$$

is a push out system. 
Proof. Suppose that $x_{1} \ominus g \in X_{1} \ominus G$. Hence

$$
\pi \circ(\varphi \ominus I)\left(x_{1} \ominus g\right)=\pi\left(\varphi\left(x_{1}\right) \ominus g\right)=\xi^{*}\left(\varphi\left(x_{1}\right) \ominus g\right)
$$

and

$$
\begin{aligned}
\beta \circ \psi\left(x_{1} \ominus g\right)=\beta\left(x_{1} \delta g\right) & =\xi^{*}\left(\varphi\left(x_{1} \delta g\right) \ominus e_{\delta}\right) \\
& =\xi^{*}\left(\varphi\left(x_{1}\right) \delta e_{\delta} \ominus g\right) \\
& =\xi^{*}\left(\varphi\left(x_{1}\right) \ominus e_{\delta} \delta g\right) \\
& =\xi^{*}\left(\varphi\left(x_{1}\right) \ominus g\right) .
\end{aligned}
$$

This implies that $\beta \circ \psi=\pi \circ(\varphi \ominus I)$. Let $T^{\prime}$ be a $(\Delta, G)$-set, $\beta^{\prime}: X_{1} \longrightarrow T^{\prime}$ and $\sigma: X_{2} \ominus G \longrightarrow T^{\prime}$ such that $\sigma \circ(\varphi \ominus I)=\beta^{\prime} \circ \psi$. For every $x_{1}, x_{1}^{\prime} \in X_{1}$ and $g, g^{\prime} \in G$ such that $\xi^{*}\left(\varphi\left(x_{1}\right) \ominus g\right)=\xi^{*}\left(\varphi\left(x_{1}^{\prime}\right) \ominus g^{\prime}\right)$. We have

$$
\begin{aligned}
\pi\left(\varphi\left(x_{1}\right) \ominus g\right)=\pi \circ(\varphi \ominus I)\left(x_{1} \ominus g\right) & =\beta^{\prime} \circ \psi\left(x_{1} \ominus g\right) \\
& =\beta^{\prime}\left(x_{1} \delta g\right) \\
& =\beta^{\prime}\left(x_{1}^{\prime} \delta g^{\prime}\right) \\
& =\psi\left(\varphi\left(x_{1}^{\prime}\right) \ominus g^{\prime}\right) .
\end{aligned}
$$

It follows that $\psi$ induces a unique morphism $\omega: T \longrightarrow T^{\prime}$ by following definition:

$$
\omega\left(\xi^{*}\left(x_{2} \ominus g\right)\right)=\pi\left(x_{2} \ominus g\right) .
$$

On the others hand

$$
\begin{aligned}
\omega \circ \beta\left(x_{1}\right)=\omega\left(\xi^{*}\left(\varphi\left(x_{1}\right) \ominus e_{\delta}\right)\right) & =\pi \circ\left(\varphi\left(x_{1}\right) \ominus e_{\delta}\right) \\
& =\pi \circ(\varphi \ominus I)\left(x_{1} \ominus e_{\delta}\right) \\
& =\beta^{\prime} \circ \psi\left(x_{1} \ominus e_{\delta}\right) \\
& =\beta^{\prime}\left(x_{1}\right) .
\end{aligned}
$$

This completes the proof.

Lemma 3.1. Let $G_{1}$ be a $\Gamma$-subsemihypergroup of $G$ and $G_{1}$ has the extension property in $G$ and $\varphi: X_{1} \longrightarrow X_{2}$ be a morphism on $G_{1}$ and $x_{2} \ominus e_{\alpha}=\varphi\left(x_{1}\right) \ominus g$. Then, $x_{2} \in \operatorname{Im} \varphi$.

Proof. Suppose that $X_{1}, X_{2}, T$ are $(\Delta, G)$-sets and $\varphi: X_{1} \longrightarrow X_{2}, \delta_{2}: X_{2} \longrightarrow T$ and $\lambda: X_{2} \longrightarrow T$ are a push out system. Hence $X_{1} \ominus G, X_{2} \ominus G, T \ominus G, \varphi \ominus I_{G}$ : $X_{1} \ominus G \longrightarrow X_{2} \ominus G, \lambda \ominus I_{G}: X_{2} \ominus G \longrightarrow T \ominus G$ and $\delta_{2} \ominus I_{G}: X_{2} \ominus G \longrightarrow T \ominus G$ is 
also push out system. Since $x_{2} \ominus e_{\alpha}=\varphi\left(x_{1}\right) \ominus g$ in $X_{2} \ominus G$, we have

$$
\begin{aligned}
\delta_{2}\left(x_{2}\right) \ominus e_{\alpha}=\left(\delta_{2} \ominus I_{G}\right)\left(x_{2} \ominus e_{\alpha}\right) & =\left(\delta_{2} \ominus I_{G}\right)\left(\varphi\left(x_{1}\right) \ominus g\right) \\
& =\delta_{2}\left(\varphi\left(x_{1}\right)\right) \ominus g \\
& =\lambda\left(\varphi\left(x_{1}\right)\right) \ominus g \\
& =\left(\lambda \ominus I_{G}\right)\left(\varphi\left(x_{1}\right) \ominus g\right) \\
& =\left(\lambda \ominus I_{G}\right)\left(x_{2} \ominus e_{\alpha}\right) \\
& =\lambda\left(x_{2}\right) \ominus e_{\alpha} .
\end{aligned}
$$

Since $G_{1}$ has the extension property in $G$ the map $X_{2} \longrightarrow X_{2} \ominus e_{\alpha}$ from $X_{2}$ to $X_{2} \ominus G$ is one to one. This implies that $\lambda\left(x_{2}\right)=\delta_{2}\left(x_{2}\right)$ and by Proposition 3.4, $x_{2} \in \operatorname{Im} \varphi$.

Theorem 3.2. Let $G_{1}$ be a $\Gamma$-subsemihypergroup of $G$ and $G_{1}$ has the extension property in $G$ and $\varphi: X_{1} \longrightarrow X_{2}$ be a morphism on $G_{1}$ such that $\varphi \ominus I: X_{1} \ominus X \longrightarrow$ $X_{2} \ominus X$ be a morphism, where $X$ be a right $(\Delta, G)$-set. Then, $x_{2} \ominus e_{\alpha} \ominus x=\varphi(x) \ominus g \ominus x^{\prime}$, implies that there exist $t_{1} \in X_{1}, t_{2} \in X$ such that $x_{2} \ominus e_{\alpha} \ominus x=\varphi\left(t_{1}\right) \ominus e_{\alpha} \ominus t_{2}$.

Proof. Suppose that $x_{2} \ominus e_{\alpha} \ominus x=\varphi(x) \ominus g \ominus x^{\prime}$. Let $X_{1}, X_{2}, T$ be push out system, where $\varphi: X_{1} \longrightarrow X_{2}, \delta_{1}: X_{2} \longrightarrow T$ and $\delta_{2}: X_{2} \longrightarrow T$. Hence $X_{1} \ominus X, X_{2} \ominus X$, and $T \ominus X$ is a push out system, where $\varphi \ominus I: X_{1} \ominus X \longrightarrow X_{2} \ominus X, \delta_{1} \ominus I: X_{2} \ominus X \longrightarrow T \ominus X$ and $\delta_{2} \ominus I: X_{2} \ominus X \longrightarrow T \ominus X$ is also push out system. On the other hand on $T \ominus G \ominus X$,

$$
\begin{aligned}
\left.\delta_{2}\left(x_{2}\right) \ominus e_{\alpha} \ominus x=\left(\delta_{2} \ominus I_{G} \ominus I_{X}\right)\left(x_{2} \ominus e_{\alpha} \ominus x\right)\right) & =\left(\delta_{2} \ominus I_{G} \ominus I_{X}\right)\left(\varphi(x) \ominus g \ominus x^{\prime}\right) \\
& =\delta_{2} \varphi(x) \ominus g \ominus x^{\prime} \\
& =\delta_{2} \varphi(x) \ominus g \ominus x^{\prime} \\
& =\delta_{1} \varphi(x) \ominus g \ominus x^{\prime} \\
& =\delta_{1}\left(x_{2}\right) \ominus e_{\alpha} \ominus x .
\end{aligned}
$$

By extension property we have $\delta_{2}\left(x_{2}\right) \ominus x=\delta_{1}\left(x_{2}\right) \ominus x$. Hence by Proposition 3.4 there exist $t_{1} \in X_{1}$ and $t_{2} \in X$ such that

$$
x_{2} \ominus x=\left(\varphi \ominus I_{X}\right)\left(t_{1} \oslash t_{2}\right)=\varphi\left(t_{1}\right) \oslash t_{2} .
$$

This implies that

$$
x_{2} \ominus e_{\alpha} x=\varphi\left(t_{1}\right) \ominus e_{\alpha} \ominus t_{2} .
$$

\section{REFERENCES}

[1] S. M. Anvariyeh, S. Mirvakili and B. Davvaz, On $\Gamma$-hyperideals in $\Gamma$-semihypergroups, Carpathian J. Math. 26 (2010) 11-23.

[2] P. Corsini and V. Leoreanu, Applications of Hyperstructure Theory, Kluwer Academic Publishers, Dordrecht, 2003.

[3] P. Corsini, Prolegomena of Hypergroup Theory, Second Edition, Aviani Editore, 1993.

[4] B. Davvaz and V. Leoreanu, Hyperstructure Theory and Applications, International Academic Press, 2007. 
[5] S. O. Dehkordi and B. Davvaz, A strong regular relation on $\Gamma$-semihyperrings, J. Sci. I.R. Iran. 22 (3) (2011), 257-266.

[6] S. O. Dehkordi, and B. Davvaz, Г-semihyperrings: approximations and rough ideals, Bull. Malays. Math. Sci. Soc. 35 (4) (2012), 1035-1047.

[7] S. Ostadhadi-dehkordi, Twist product derived from $\Gamma$-semihypergroup, submitted for publication.

[8] D. Heidari, S. O. Dehkordi and B. Davvaz $\Gamma$-semihypergroups and their properties, U.P.B. Sci. Bull., Series A, 72 (1) (2010), 195-208.

[9] F. Marty, Sur une generalization de la notion de group, Proceedings of the 8th Congres des Mathematiciens Scandinave, Stockholm, Sweden, 1934, pp. 45-49.

Department of Mathematics,

HORMOZGAN UNIVERSITY,

BANDAR ABBAS, IRAN

E-mail address: Ostadhadi-Dehkordi@hotmail.com, Ostadhadi@hormozgan.ac.ir 